\title{
Cognitive deficit related to seizure pattern in centrencephalic epilepsy
}

\author{
DOREEN KIMURA ${ }^{1}$
}

\author{
From Montreal Neurological Institute
}

The identifying characteristics of the electroencephalogram in centrencephalic epilepsy have been outlined by Penfield and Jasper (1954) as including a bilaterally synchronous onset of abnormality which consists of wave-and-spike activity at or near a frequency of 3 per second. This is in contrast to the E.E.G. abnormality seen in cases of focal cortical epilepsy in which sharp waves predominate, begin in a restricted area, and spread either slowly or not at all to other parts of the brain. The seizures in these two types of epilepsy also differ, the centrencephalic seizures including 'absence' attacks, myoclonic jerks, akinetic attacks, and major seizures, while the attacks in focal cortical epilepsy vary with the location of the focus.

In the case of focal discharge, the E.E.G. abnormality is of course assumed to originate from this focus. In the centrencephalic type of epilepsy, however, the bilaterally synchronous onset of E.E.G. abnormality led Jasper and Kershman (1941) to believe that the origin here was in some subcortical region which had widespread connexions with cortical areas so that it could act as pacemaker for the entire cortex. This interpretation was given strong support by an experimental study in the cat, in which stimulation of a small area in the medial thalamus evoked widespread bilaterally synchronous 3 per second spike-and-wave activity in the cortex (Jasper and Fortuyn, 1947). Williams (1953), recording from children with petit mal attacks, confirmed the important role of the thalamus in the production of pathological spike-and-wave activity.

Since the abnormalities in focal and non-focal epilepsy apparently derive from different anatomical loci, it is reasonable to suppose that they may give rise to different functional disturbances. Penfield and Jasper (1954) have suggested that the area involved in centrencephalic seizures plays an important role in the maintenance of a state of consciousness. Mirsky, Primac, Ajmone-Marsan, Rosvold, and Stevens (1960) have made a direct comparison of the performance of focal and non-focal groups on a test

${ }^{1}$ Now at St. Joseph's Hospital, Ham:lton, Canada. demanding sustained attention (continuous performance test). They found that the patients with centrencephalic epilepsy were impaired whereas those with focal cortical epilepsy were not. On memory tests, however, the centrencephalic group performed better than a group of patients with bitemporal epilepsy, indicating some specificity of dysfunction.

The present study provides further support for such a functional differentiation, and at the same time suggests a further differentiation based upon a division of the centrencephalic group into two subgroups.

\section{METHOD}

SUBJECTS All subjects were patients at the Montreal Neurological Institute. They had had epileptic seizures for, on the average, from 10 to 15 years, and they had been admitted for possible surgery for the removal of epileptogenic tissue. Patients classified as centrencephalic did not undergo surgery, and the data reported here for the focal cases are pre-operative data.

The centrencephalic group fulfilled the following criteria:-1 The diagnosis on discharge was centrencephalic epilepsy. 2 There were no seizures with unilateral onset in one limb (only one patient was excluded in this way). 3 The E.E.G. records showed bilaterally synchronous abnormality with spike-and-wave or multiple spikeand-wave between 2 and 4 per second.

Patients with focal epilepsy were classified into four groups on the basis of focus of epileptic activity. Most of these patients subsequently underwent surgery. The four groups are: left temporal, right temporal, bitemporal, and unilateral frontal. Age and intelligence data for each group are given in Table I.

TABLE I

AGE AND INTELLIGENCE DATA

\begin{tabular}{llr} 
Group & Age & I.Q. \\
\hline Centrencephalic & $19 \cdot 4$ & $101 \cdot 0$ \\
Left temporal & $31 \cdot 2$ & $110 \cdot 7$ \\
Right temporal & $30 \cdot 3$ & $110 \cdot 4$ \\
Unilateral frontal & 23.9 & $107 \cdot 0$ \\
Bitemporal & 34.9 & $98 \cdot 5$
\end{tabular}


PROCEDURE All subiects were given a variety of perceptual and memory tests which will not be described here. The test to be described in detail is a letter-ordering task. There were two phases to this task. In the first phase, four letters forming a horizontal row, two on either side of the fixation point, were flashed on a screen by means of a tachistoscope. The subject had simply to report the letters he saw. His responses, as well as his response time, were recorded. After 10 such presentations, the second phase of the task began. The procedure was the same, except that now the subject was required to report the letters in alphabetical order. Thus, if he saw GTKD he was to say DGKT. The recording and timing of responses were done from behind the tachistoscope, and care was taken to conceal from the subject the fact that he was being timed. Mistakes in ordering were strongly discouraged, so that the main measure here (aside from accuracy in identifying the letters) was the average time required to accomplish the ordering.

\section{RESULTS}

The mean ordering time for each group is given in Table II. It is clear that the centrencephalic group takes longer to order letters than any cther group. When these data are subjected to analysis of variance the $\mathrm{F}$ ratio is $3.73(\mathrm{P}<0.01)$ and subsequent $\mathrm{t}$ tests show a significant difference between the centrencephalic group and every other group $(P<0.02$ for the least significant difference). There are no differences among the other groups. That this effect is not due simply to slow reaction time can be seen by looking at the simple response time for saying the letters, from the first phase of the task. This is also shown in Table II. There are no differences among groups in simple response time. There are also no differences among groups in the accuracy with which letters are reported.

TABLE II

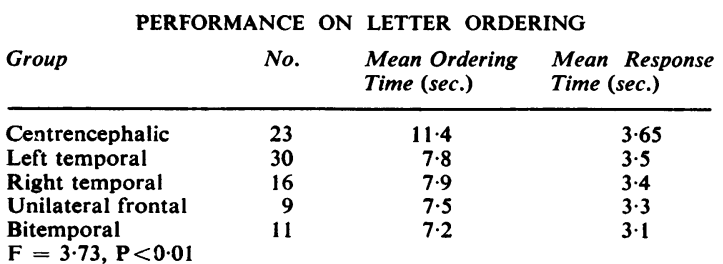

There are of course many factors which differentiate the centrencephalic group from other groups, besides the one related to origin of discharge. A detailed study was made of the relation between each of age, medication, and frequency of seizures on the one hand, and ordering time on the other, and the results are discussed below. None of these factors was satisfactorily related to ordering time, and a closer study was therefore made within the centren- cephalic group of any features which might influence performance. It soon appeared that those patients whose seizure history included the occurrence of myoclonic jerks were not impaired on this task? The comparison between the two subgroups is give in Table IIl.

\section{TABLE III}

LETTER ORDERING IN TWO CENTRENCEPHALIC GROUPS

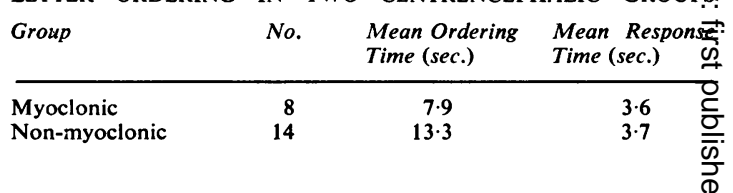

The myoclonic group is comparable to the focas groups in Table II in ordering time, while the difficulty with ordering in the remaining non-myo $\vec{\sigma}$ clonic group is enhanced after exclusion of the eight myoclonic patients. (One subject was dropped be cause the occurrence of myoclonic jerks was question able.) When an analysis of variance was performe with these two centrencephalic groups and the fouis focal groups, the $F$ ratio is $4.69(\mathrm{P}<0.001)$, and the difference between the two centrencephalic stgbio groups is significant $(\mathbf{P}<\mathbf{0 . 0 1})$. Again, there are differences in simple response time.

A second task apparently sensitive to centrenee. phalic disturbance was begun much later in series of patients, so that statistical comparison is yet profitable. In this test, a modification of Knox cube test, five black blocks are placed in a between experimenter and subject. $\mathrm{E}$ taps the block\$ in a particular order and $S$ simply taps them subsequently in the same order. The score is the number of correct trials out of 10 . Nine patients from the non-myoclonic group have been tested and obtaine $\bar{\Phi}$ a mean score of $5 \cdot 1$. Five patients from the myog clonic group obtained a mean score of $7 \cdot 2$, which wa@ again comparable to the scores obtained by th $\overrightarrow{\vec{E}}$ focal groups. Thus it appears that on this test alsळ the non-myoclonic group is inferior to the myoz clonic. It should be emphasized that the two groups do not differ in I.Q., nor in their performance on variety of perceptual and memory tasks.

RELATION OF VARIOUS FACTORS TO PERFORMANC? The centrencephalic group as a whole is younge? than the other groups. However, there is only a sma insignificant correlation between age and ordering time, and it is moreover a positive one. It is thus obvious that age plays no part in the deficit present in the centrencephalic group. Also intensively studief: was the effect on ordering of medication. Though the details cannot be given, there appeared to be no res lation between ordering time and type of drug taken 
in the month preceding testing. Contrary to expectation, there was no systematic difference between medication administered to centrencephalic and focal groups before admission. While in hospital and during testing, patients were usually not on medication. Thus medication does not influence these data. This is not to say, of course, that in normal subjects the acute administration of drugs will not influence the ordering task, since Mirsky, Primac, and Bates (1959) found certain drugs to have an effect on the continuous performance test, which is also sensitive to centrencephalic abnormality.

Table IV gives a summary of the seizure history of these patients. These are very crude estimates taken from the patients' records, themselves based on estimates made by the immediate family. They do, however, permit the conclusion that there is no systematic relation between ordering time and either the duration of illness or the frequency of major seizures. The non-myoclonic group has more minor seizures per day than the focal groups, but this is true for the myoclonic group as well. Frequency of minor seizures per se thus does not account for the slowness in ordering time. Indeed, the factors which are related to deficiency on this task can best be studied by comparing the two centrencephalic subgroups.

\section{TABLE IV}

SEIZURE HISTORY

\begin{tabular}{lcccccc} 
Group & $\begin{array}{l}\text { Minor } \\
\text { per } \\
\text { Day }\end{array}$ & Range & $\begin{array}{l}\text { Major } \\
\text { per } \\
\text { Month }\end{array}$ & Range & $\begin{array}{l}\text { Years } \\
\text { since } \\
\text { Onset }\end{array}$ & Range \\
\hline Myoclonic & $8 \cdot 0$ & $0-50$ & $0 \cdot 55$ & $0-1$ & $7 \cdot 25$ & $3-12$ \\
Non-myoclonic & $4 \cdot 8$ & $0-20$ & $2 \cdot 9$ & $0-20$ & $9 \cdot 6$ & $1-35$ \\
Unilateral temporal & $1 \cdot 3$ & $0-10$ & $1 \cdot 7$ & $0-20$ & $17 \cdot 5$ & $7-31$ \\
Unilateral frontal & 0.9 & $0-3$ & $4 \cdot 5$ & $0-30$ & $10 \cdot 8$ & $2-28$ \\
Bitemporal & $0 \cdot 5$ & $0-3$ & $1 \cdot 4$ & $0-7$ & $15 \cdot 1$ & $2-31$
\end{tabular}

FACTORS WITHIN THE CENTRENCEPHALIC GROUP All patients in the myoclonic group had spontaneous myoclonic jerks, i.e., no patients were included in this group who had sucil jerks only during photic stimulation. Of the eight patients in the myoclonic group, six were known to have major seizures as well, one had questionable absence attacks, and one had a variety of seizures including tonic and akinetic attacks. The jerks reported by the family or observed in hospital were synergic, i.e., involving at least a whole limb, and usually bilateral and synchronous. They were thus not strictly 'myoclonic' at all but rather of more central origin (Krebs, 1952). The group corresponds to the sporadic or intermittent type of myoclonic epilepsy described by Lundborg, not to 'myoclonus epilepsy', a progressive degenerative disease (see Lennox and Lennox, 1960).

Of the 14 patients in the non-myoclonic group, seven had only 'absences' and major seizures. Three more had absences and major attacks with other types of seizure. The remaining four patients had a variety of one or more types of seizure, including absence or major seizures, tonic stiffening, running attacks, head turning (no consistent lateralization), and akinetic attacks. It was expected that a relationship might be found between the occurrence of absence attacks and slowness on the ordering task, but no consistent relation was found. Thus four patients with classical absence and major attacks were not particularly slowed on the ordering task, whereas one patient with head turning, urinary incontinence, and major seizures, and another who had only running fits, were markedly slow on ordering. The number of patients within the non-myoclonic group with each type of seizure is of course small, so that no firm conclusions can be drawn, but the data so far do not suggest that slowness on ordering occurs more reliably in patients with absence attacks.

Slowness on ordering is not an artifact of the occurrence of overt seizures, for ordering time was never recorded for any occasion on which a seizure occurred. Instead, a new trial would be given. Moreover, the myoclonic group has as many seizures per day as the non-myoclonic group, so that one cannot credit even the indirect effects of having many seizures with producing impairment on this task. Since the two groups do not differ in frequency of clinical seizures, it seems unlikely that they differ with respect to subclinical seizures, i.e., in the frequency of abnormal E.E.G. bursts not accompanied by overt seizures. Nevertheless, since E.E.G. records were not taken during testing, the possibility cannot be ruled out. There are, however, several objections to this viewpoint as an explanation for the difference on ordering. 1 Disruption of behaviour is much less likely during subclinical bursts than during clinical seizures (Davidoff and Johnson, 1964). 2 There are no apparent differences in E.E.G. abnormality, as judged from clinical E.E.G. reports, between myoclonic and non-myoclonic groups in this study, that is, there are no differences in the incidence of waveand-spike abnormality, in the basic frequencies in which it appears, or in sensitivity to photic stimulation or hyperventilation. The differences outlined by Penfield and Jasper (1954) between myoclonic and petit mal wave-and-spike characteristics do not appear here, possibly because the non-myoclonic group is so mixed. 3 If slowness on ordering were due to occasional subclinical bursts one would expect the subjects to perform normally a good part of the time, and only occasionally to have very long ordering times. Of the patients in the non-myoclonic group who are slow on ordering (average more than $10 \mathrm{sec}$.) slightly over half of them rarely perform ordering 
in less than $10 \mathrm{sec}$, i.e., they are consistently slow. This does not accord well with a picture of occasional interference.

\section{DISCUSSION}

ANATOMICAL CONSIDERATIONS It is by now well established that bursts of spike-and-wave activity are often related to impaired performance on certain tasks, though the relation between such abnormal bursts and degree of impairment is by no means simple (Davidoff and Johnson, 1964; Mirsky and Van Buren, 1962). More interesting is the fact that similar impairment exists in the intervals between bursts of abnormality. Thus Mirsky and Van Buren found that the centrencephalic group was still significantly inferior to focal groups even when no abnormal activity appeared in the E.E.G. The difference found in the present study between patients with a history of myoclonic jerks and those without can perhaps also best be understood as a function, not only of abnormality reflected in the E.E.G., but of the different subcortical circuits involved in the two types of disorder.

Penfield and Jasper (1954) have suggested that myoclonic attacks, as opposed to absence attacks, may be initiated by subcortical centres which are not involved in the maintenance of consciousness. It is commonly accepted that the synergicjerks result from a sudden group discharge to lower motor neurons, and that some area in the lower brain-stem plays an important part in these sudden discharges. Carels (1960), in considering the anatomical lesions found in many kinds of myoclonus, reports that the most frequent sites are the brain-stem and the cerebellum. He suggests that the reticular formation is the preponderant participant in the production of myoclonic jerks, perhaps through the presence there of an irritative lesion, but that other structures, such as the cerebellum, may also initiate such jerks. It is beyond the scope of this paper to discuss in detail all the possible circuits for the initiation of myoclonic jerks. The manner in which the cerebellum might act on the reticular formation to produce them is outlined by Gozzano and Viziolo (1959).

It is possible, then, that the reticular formation may in some way be involved in patients who have myoclonic jerks. On the other hand, the area most probably associated with the genesis of petit mal attacks is the more rostral non-specific thalamic system (Jasper and Fortuyn, 1947). For the present we will assume that the other types of seizures present in the non-myoclonic group are also closely involved with this system, or at least that they have more in common with it than with the myoclonic system.

The difficulty on the ordering task may thus reflect a chronic dysfunction of the more rostral 'attentione system, or of other structures which normally in $z$ fluence it. This dysfunction would be present eve when there is no electroencephalographic abnoro mality, but it might be exaggerated during wave-and spike bursts. The myoclonic system, acting through the rostral system, might also initiate wave-and-spike activity, sometimes associated with momentars disruption of attention. Between bursts, however there need be no dysfunction of attention.

NATURE OF THE DEFICIT The assumption made throughout this discussion has been that the impaire ment on the tests described here is a deficit in the capacity for sustained concentration, described by Mirsky et al. (1960) as a deficit in attentiveness. OR the ordering task this deficit would be reflected inf the longer time required to perform the task correctly $\overrightarrow{0}$ whereas on the block-tapping test it would be re:flected in lower accuracy scores. However, there i $\overrightarrow{\&}$ another factor involved in these tests apart from the degree of concentration required: this is the fact that they are serial tasks. The subject must keep in min more than one event at a time and respond to these events in a sequential way. Milner (1961) has prop posed a neural mechanism for the immediate rechlo of sequences which might involve cells of the nom specific projection systems. It may be that serge tasks are simply more sensitive to lowered alertnegs than are other types of tasks, but at present cannot rule out the possibility that it is the seriaE nature of the task which reveals the deficit.

\section{SUMMARY}

A test in which letters presented randomly had to be reported alphabetically was given to patients with various types of epilepsy. Ordering time was found to be slower for patients with centrencephalic epilepsyo than for patients with focal cortical epilepsy. Within the centrencephalic group, however, patients with myoclonic jerks were found to be unimpaired. The results are discussed in terms of the subcortica circuits which may be involved in the capacity for sustained concentration.

This research was supported by grant B-2831 to $\mathrm{Dr}_{\overline{0}}^{\overline{0}}$ Brenda Milner from the U.S. Public Health Service andB by grant 9401-11 to Dr. D. O. Hebb from the Defencos Research Board, Ottawa. Support during the writing of the paper was provided by a grant from Geigy-Jubilaeums Stiftung, Basle. I am grateful to Dr. Milner for suggestions throughout the investigation.

\section{REFERENCES}

Carels, G. (1960). Wld Neurol., 1, 524-534.

Davidoff, R. A., and Johnson, L. C. (1964). Electroenceph. Clin Neurophysiol., 16, 343-354. 
Gozzano, M., and Vizioli, R. (1959). Int. J. Neurol. (Montevideo), $1,34-44$.

Jasper, H., and Drocgleever-Fortayn, J. (1947). Res. Publ. Ass. Res. nerv. Dis., 26, 272-298.

- and Kershman, J. (1941). Arch. Neurol. Psychiat. (Chic.), 45, 903-943.

Krebs, E. (1952), Rev. neurol., 86, 549-566.

Lennox, W. G., and Lennox, M. A. (1960). Epilepsy and Related Disorders. Little, Brown, Boston.
Milner, P. M. (1961). Kybernetik, 1, 76-81.

Mirsky, A. F., Primac, D. W., Ajmone Marsan, C., Rosvold, H. E., and Stevens, J. R. (1960). Exp. Neurol., 2, 75-89. - and Bates, R. (1959). J. nerv. ment. Dis., 128, 12-17.

-, and Van Buren, J. (1962). Paper read to American Epilepsy Society, New York.

Penfield, W., and Jasper, H. (1954). Epilepsy and the Functional Anatomy of the Human Brain. Little, Brown, Boston. Williams, D. (1953). Brain, 76, 50-69. 